\title{
ANALYSIS OF INHOMOGENEITY OF MECHANICAL PROPERTIES OF HOT ROLLED AND COLD STRETCHED RIBBED WIRE
}

\author{
1,2Richard FABÍK, ${ }^{1}$ Jakub MOLNÁR, ${ }^{2}$ Tomáš SANTARIUS \\ ${ }^{1}$ VSB - Technical University of Ostrava, Ostrava, Czech Republic, EU \\ ${ }^{2}$ Liberty Ostrava a.s., Ostrava, Czech Republic, EU, richard.fabik@libertysteelgroup.com
}

https://doi.org/10.37904/metal.2019.690

\begin{abstract}
The paper deal with experience with the introduction of production of ribbed wire from B500B steel on the cold stretching line $\mathrm{KOCH}$ in ArcelorMittal Ostrava. The basic principle of work hardening during cold stretching is introduced. Distribution of mechanical properties along the wire length of both hot rolled and cold stretched wire were performed. Statistical analysis has shown that there is a definable cause affecting the manufacturing process. Because of this, the yield strength, tensile stress and elongation to fracture values showing a trend along the wire length. It has been proven by measuring of the temperature of the wire at the conveyor by thermovision, that cooling of the wire rod on the chain conveyor is this definable cause. Different parts of the wire are cooled with varying intensity due to different densities of wire loops stacking on the conveyor. This fact directly affects the mechanical properties of the wire. Higher cooling rates result in higher yield strength and tensile stress values and lower elongation to fracture values. The effect of cold stretching on the change in the trend of mechanical properties over the length of the wire was also investigated. Interestingly, the variability of mechanical properties after cold stretching has increased.
\end{abstract}

Keywords: Cold stretching, ribbed steel wire, B500B, mechanical properties

\section{INTRODUCTION}

Nowadays for producing of ribbed wire with superior mechanical properties like B500B, B550B are use these technologies [1]:

- Thermomechanical rolling and control cooling,

- $\quad$ Quenching and self tempering (QST) immediately after hot rolling,

- Hot rolling of round wire and cold rolling of ribbed wire,

- Hot rolling of ribbed wire and cold stretching (CS).

First two technologies improve both yield strength and ductility. On the other hand, last two technologies increased yield strength but decreased ductility due to work hardening $[1,2]$.

ArcelorMittal Ostrava produce ribbed bars on Medium and Fine rolling mill by QST and ribbed wire by CS on Wire rod mill and CS line $\mathrm{KOCH}$. During CS process the wire is continuously stretched between bending pulleys and so-called S-roller (Figures 1 and 2).

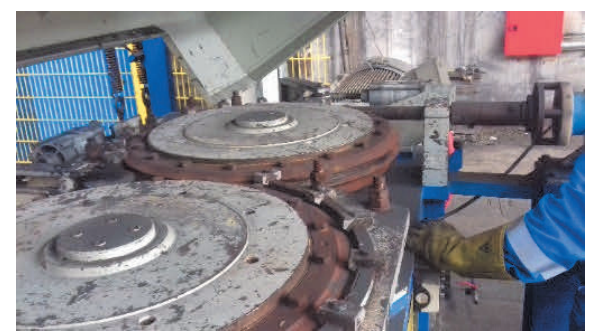

Figure $1 \mathrm{KOCH}$ line: S-roller

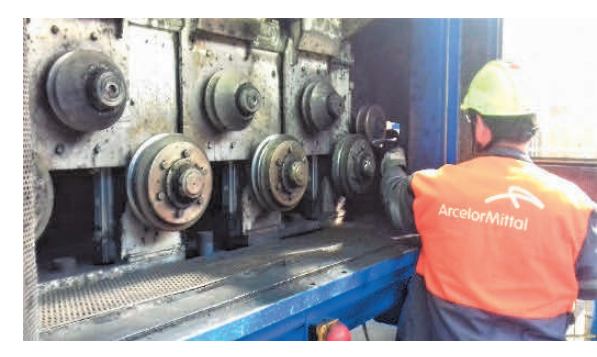

Figure $2 \mathrm{KOCH}$ line: Bending pulleys 
The value of strain can be controlled by shifting of bending pulleys up and down. Typical influence of longitudinal deformation on shifting of bending pulleys is represented on the graph on Figure 3. Deformation values too fluctuate around the mean value, so from the practical point of view it is better to use shifting of bending pulleys to express deformation.

To ensure that final wire match the standard requirements [3] is necessary analyse how the CS process influenced on mechanical properties and geometry of wire. In Figure $\mathbf{4}$ we can see relationship between properties of final wire and CS process schematically. Most important thing is balance between Yield strength and Elongation at maximal load. We can obtain elongation of the wire during CS in range $2-5 \%$. This deformation

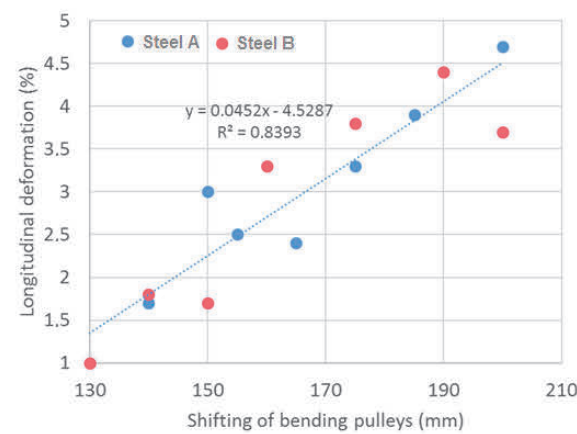

Figure 3 Influence of shifting of bending pulleys on longitudinal deformation causes increasing of Yield strength about 110 to $140 \mathrm{MPa}$ (minimum according DIN488 standard for wire in coils is $525 \mathrm{MPa}$ ), but also decreasing of Elongation at maximum load about 4.5 to 6.5 percentage points (minimum according DIN488 standard for wire in coils is $5.9 \%$ ). Also others parameters are changed during cold stretching. Weight per meter is decreased (linearly by up to $5 \%$ ), but if we produce the wire in plus tolerances, we can obtain up to $20 \mathrm{MPa}$ on Yield strength additionally (the Yield strength is related to the nominal diameter of the wire). Influence of CS on most important geometrical parameter as it is relative rib area is statistically negligible.

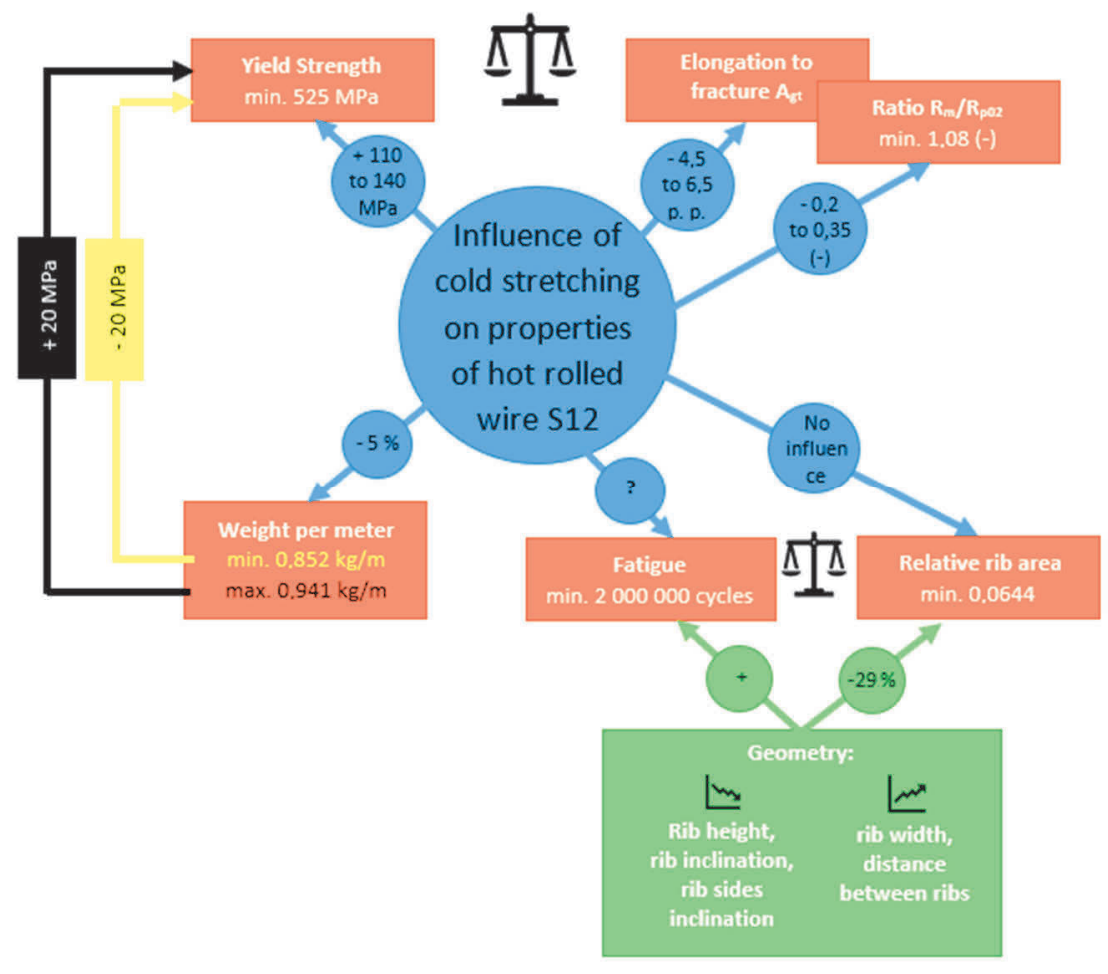

Figure 4 Schematic illustration of influence of cold stretching on properties of final ribbed wire

As stated above, the balance between Yield strength and Elongation at maximal load is critical. Based on our experience [4] with commissioning of $\mathrm{KOCH}$ line and from process capability point of view, the usable deformation range is highly influenced by standard deviations of measured data. This paper deal with analysis of causes of non-homogeneity of mechanical properties of ribbed wire from B500B steel after hot rolling and after cold stretching. 


\section{EXPERIMENTAL PART}

Experimental coils from B500B steel grade were rolled at wire rod mill (WRM) and cold stretched on $\mathrm{KOCH}$ line in ArcelorMittal Ostrava (AMO). This specific combination of obsolete rolling mill and state of the art CSline has influence on final properties of ribbed wire at unique way. In particular, the cooling of the wire after rolling on chain conveyor is a major source of variability of mechanical properties due to nonhomogeneous cooling of laying loops. There is natural cooling of wire on chain conveyor only which leads to a different cooling rate depending on the loop density.

To analyzed inhomogeneity of mechanical properties both hot rolled and cold stretched wire, we took of specimens one by one from the middle part of coil (this part of coil represents steady state from the rolling and cooling on chain conveyor point of view). After hot rolling we obtained 30 specimens (at a length of single one $35 \mathrm{~cm}$ it represents approx. 10 meters of the wire), and after CS we obtained 18 specimens (at a length of single one $35 \mathrm{~cm}$ it represents approx. 6 meters of the wire). After that, we measured Yield strength, Ultimate tensile stress and Elongation at maximal load by tensile tests.

Additionally, to analyzed of cooling process on chain conveyor the temperature field of wire was measured by thermovision.

\section{RESULTS AND DISCUSSION}

\subsection{Statistical analysis of mechanical properties}

Measured values of yield strength, ultimate tensile stress and elongation at maximal load were plotted against wire length (Figures 5 and 6). Then we made analysis of data trend to decide whether the results are influenced by any definable cause or whether the variance of the data is natural, it is mean due to an indefinable cause. On the first side it is look like data has periodic trend. We use sine $(Y, U T S)$ resp. cosine $\left(A_{g t}\right)$ trendline, whose period is given by length of individual thread of wire, shift on the $y$-axis is given by average value of each variable, magnitude is given by standard deviation of each variable and shift on the $x$-axis was determined to best fit all three series of measured values. Both trends fit data well only in range of four periods. Increasing difference between trend and data is given by fact, that the perimeter of individual thread is changing circumstantially along the wire length, thus the period of sine trend can't be constant.

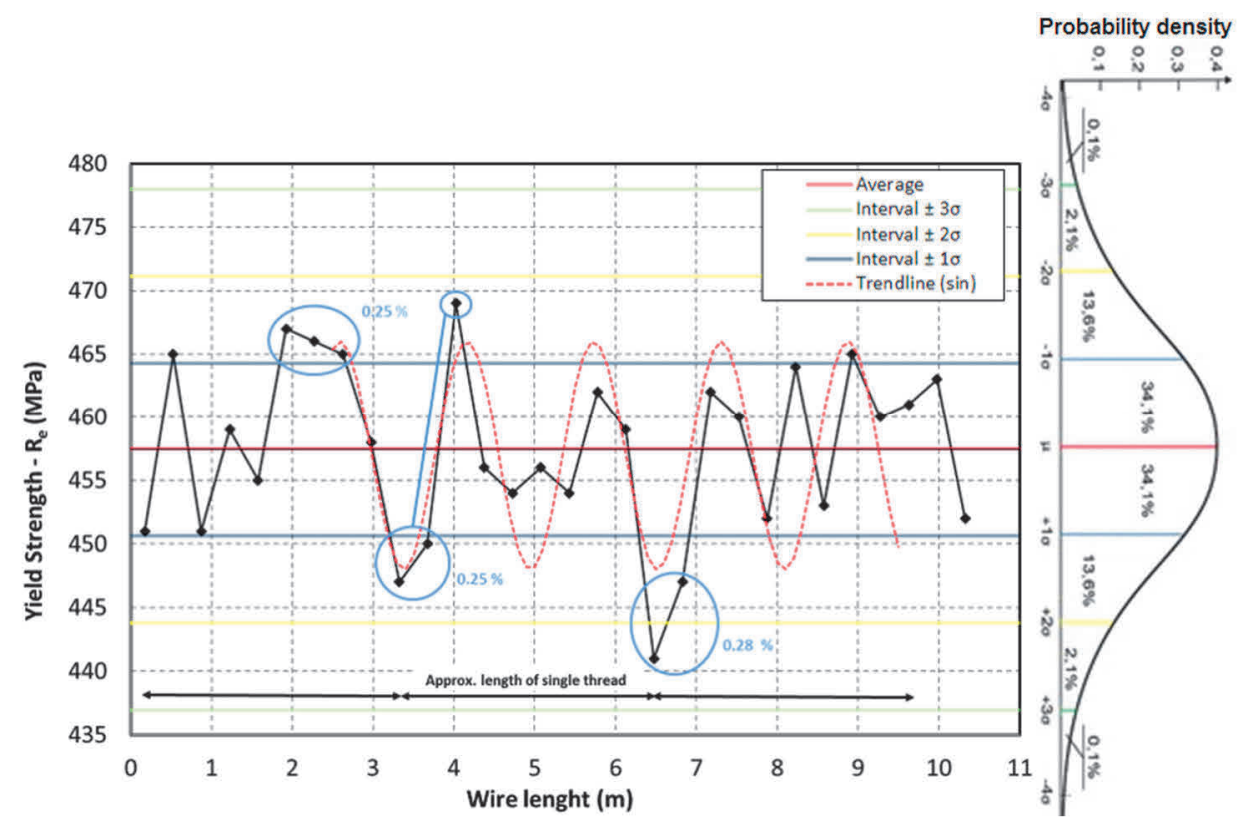

Figure 5 The measured values of yield strength of 30 specimens (black line) interleaved sinusoidal curve (dashed red). Points marked in blue indicate a low probability situation 
The more exact method of analysis uses the principles on which the control charts are based. We add in to the plot the limits that correlate with the inserted Gaussian curve and which represent the limits of intervals with different values of probability of occurrence of measured values. Now we are able to identify combinations of one by one measured values with probability lower than $0.5 \%$. Occurrence of such combinations is evidence that the process is caused by a definable cause.
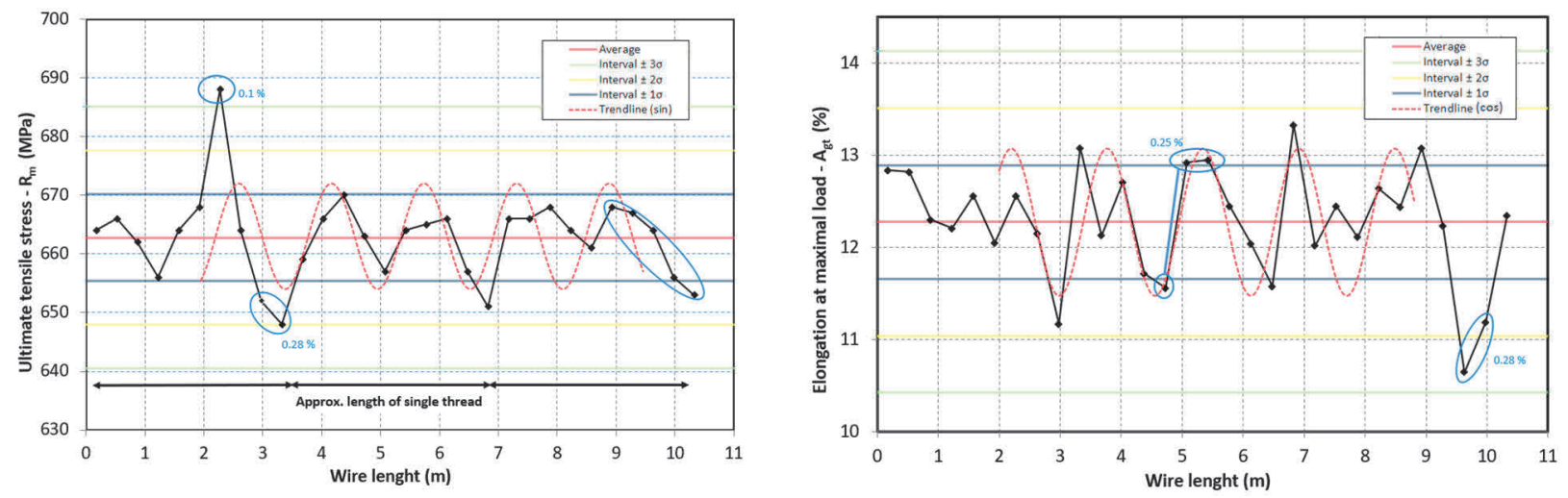

Figure 6 Measured UTS (right) and Agt (left) values from 30 test samples (black line) interspersed with sine or cosine wave (red dashed). Points marked in blue indicate a low probability situation with a computed percentage of probability.

\subsection{Relationship between cooling rate and mechanical properties}

The next step is to find a definable cause. The most important favourite is the inhomogeneous cooling of the wire on the chain conveyor.

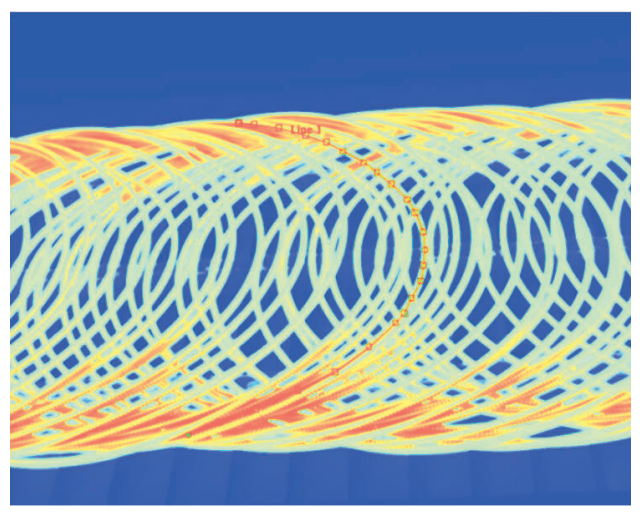

Figure 7 Temperature of wire threads laying on chain conveyor (Temperature along Line 1 see

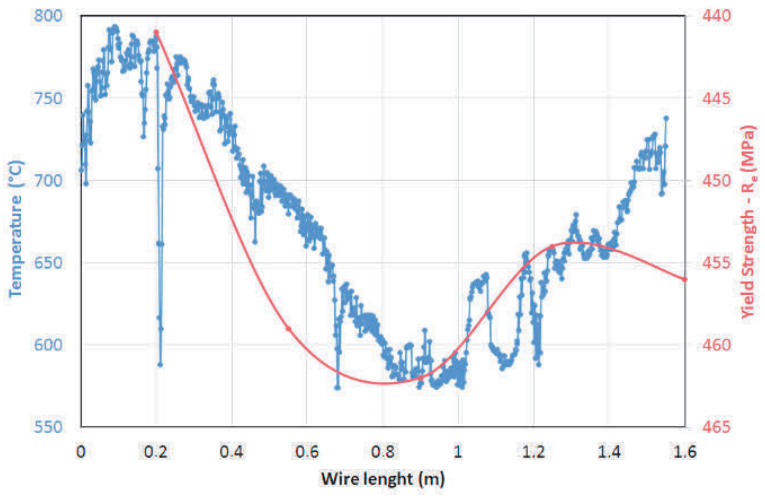

Figure 8 Superposition of temperature and measured yield strength along wire length

Figure 8)

In Figure 7 we can see the inhomogeneous distribution of the wire temperature, which is due to the different density of wire stacked on the conveyor. At the point where the wire crosses, the temperature is unambiguously higher (and thus the cooling rate is lower at that point). Assuming that the cooling rate affects the mechanical properties (lower cooling rate usually means higher strength and lower ductility), we should find a strong correlation between the temperature distribution at a particular moment on the conveyor (Figure 7) and the mechanical properties. The graph in Figure 8 shows the superposition of the temperature along one half of the thread and the measured yield strength (samples for the tensile test were not taken directly from the temperature measurement point (this is not realizable in practice), simply, we chose a part of the measured 
values from plot on Figure 5 that could correspond to the temperature distribution shown in Figure 7). The correlation between temperature and yield strength is obvious. It is apparent that the temperature on one side of the conveyor may differ substantially from the temperature on the other side of the conveyor, and this corresponds to an asymmetric yield strength curve.

\subsection{Influence of cold stretching on variability of mechanical properties}

At present, we must accept the inhomogeneity of the mechanical properties of the wire rod rolled at WRM AMO. The remaining question is how subsequent CS affects inhomogeneity of mechanical properties. The graph in Figure 9 shows the yield strength distribution along length of both hot rolled and cold stretched wire. The average yield strength was increased by $100 \mathrm{MPa}$ due to cold stretching. The standard deviation of yield strength was increased too.

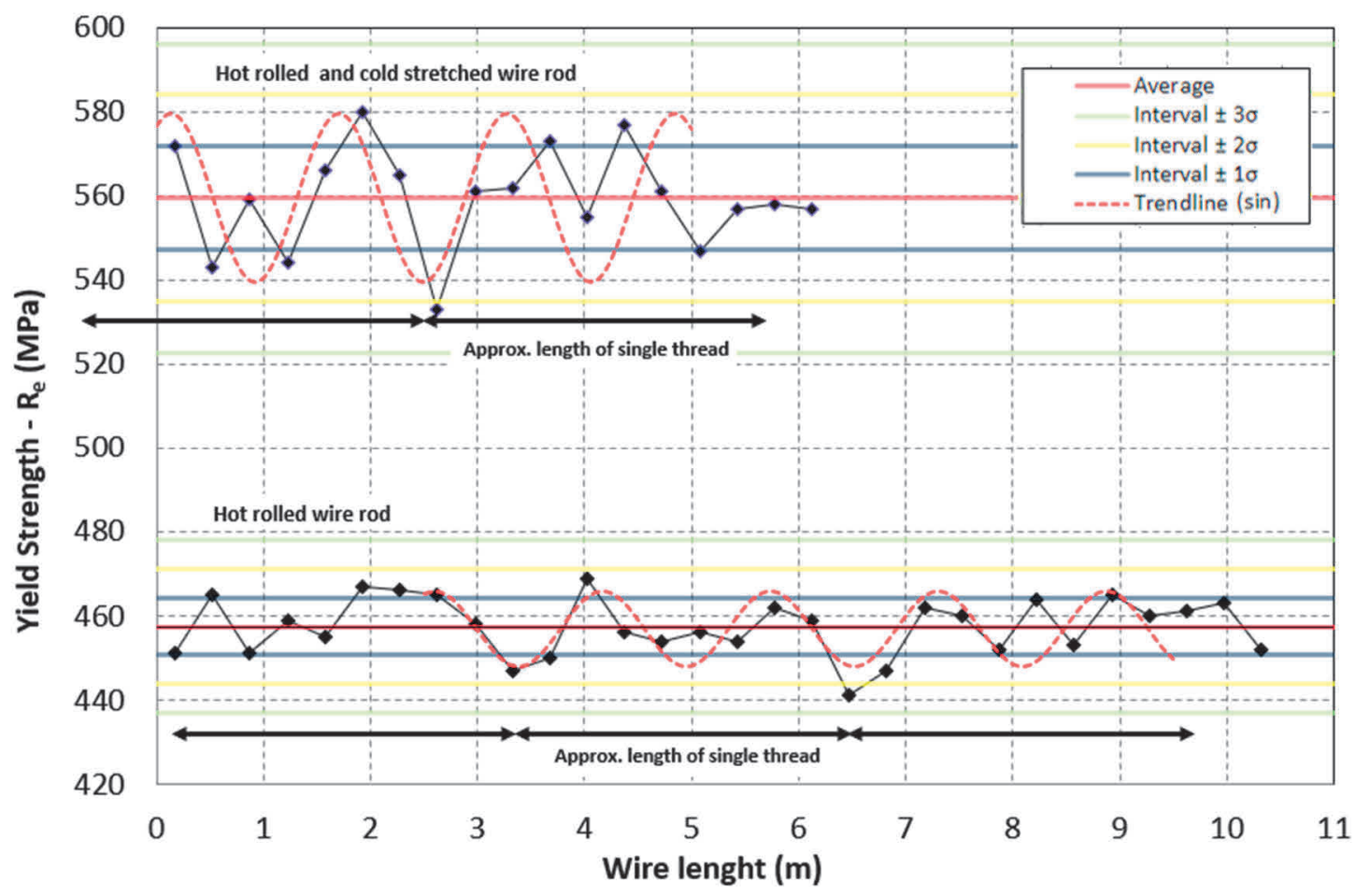

Figure 9 The measured values of yield strength before and after cold stretching

To accurately compare data variability, we use coefficient of variation which is often expressed as a percentage, and is defined as the ratio of the standard deviation $\sigma$ to the mean $\mu$. Computed coefficients of variation of measured properties of both hot rolled and cold stretched wire are listed at Table 1. If we compare the coefficients of variation before and after CS, we can see that due to CS variability of yield strength and ductility significantly increases.

Table 1 Influence of cold stretching on coefficient of variation of measured properties

\begin{tabular}{|c|c|c|c|}
\hline & \multicolumn{3}{|c|}{ Coefficient of variation (\%) } \\
\hline after: & $\mathbf{R}_{\mathbf{p} 0,2}$ & $\mathbf{R}_{\mathbf{m}}$ & $\mathbf{A g}_{\mathbf{g t}}$ \\
\hline hot rolling & 1.49 & 1.12 & 5.03 \\
\hline cold stretching & 2.19 & 1.09 & 13.12 \\
\hline difference (\%) & 46.6 & -2.6 & 160.8 \\
\hline
\end{tabular}




\section{CONCLUSION}

Based on the results presented in this paper, we can draw the following conclusions:

- Yield strength, tensile stress and elongation at maximal load values of both hot rolled and cold stretched wire showing a trend along the wire length. It has been proven that the process of hot rolling of wire rod at WRM is caused by a definable cause.

- It has been proven by measuring of the temperature, that cooling of the wire rod on the chain conveyor is this definable cause. Different parts of the wire are cooled with varying cooling rate due to different density of wire loops stacking on the conveyor. This fact directly affects the mechanical properties of the wire. Higher cooling rates result in higher yield strength and tensile stress values and lower elongation at maximal load values.

- The effect of cold stretching on the change of mechanical properties over the length of the wire was also investigated. Interestingly, the variability of mechanical properties after cold stretching has significantly increased.

- The only possible way to reduce the variability of the mechanical properties is to uniformize the cooling of the wire on the chain conveyor. This will be a very challenging task that will probably not be possible without significant investment.

\section{REFERENCES}

[1] GUO, Zhenhai. Principles of reinforced concrete. Oxford: Elsevier Butterworth-Hein, 2014. ISBN 978-0128008591.

[2] KLEMM, Michael, WAGENFUHRER, Gerhard and WAGNER, Bernhard. Der gereckte Betonstahl - ein einfaches Verfahren zur normgerechten Veredelung. Draht. 1993. Vol. 44, no. 5, pp. 263-266.

[3] DIN488-1 Reinforcing steels - Part 1: Grades, properties, marking. 2009. Normenausschuss Eisen und Stahl im DIN, Normenausschuss Bauwesen im DIN.

[4] MOLNÁR, Jakub. Analysis of non-homogeneity of mechanical properties of ribbed wire from B500B steel after hot rolling and after cold stretching. Diploma thesis. VSB-TU Ostrava. 2019. 